\title{
RETRACTED ARTICLE: Surface Defect Detection and Recognition Method for Multi-Scale Commutator Based on Deep Transfer Learning
}

\author{
Yufeng Shu ${ }^{1} \cdot$ Bin $\mathrm{Li}^{1}$
}

Received: 15 March 2021 / Accepted: 5 July 2021 / Published online: 30 July 2021

(c) King Fahd University of Petroleum \& Minerals 2021

The Editor-in-Chief and the publisher have retracted this article. The article was submitted to be part of a guest-edited issue. An investigation by the publisher found a number of articles, including this one, with a number of concerns, including but not limited to compromised editorial handling and peer review process, inappropriate or irrelevant references or not being in scope of the journal or guest-edited issue. Based on the investigation's findings the Editor-inChief therefore no longer has confidence in the results and conclusions of this article.
The authors have not responded to correspondence regarding this retraction.

Supplementary Information The online version contains supplementary material available at https://doi.org/10.1007/s13369-021-05963-3.

Springer Nature or its licensor (e.g. a society or other partner) holds exclusive rights to this article under a publishing agreement with the author(s) or other rightsholder(s); author self-archiving of the accepted manuscript version of this article is solely governed by the terms of such publishing agreement and applicable law.

Bin Li

li_bin_hust@163.com

Yufeng Shu

me.yufeng@outlook.com

1 State Key Laboratory of Digital Manufacturing Equipment and Technology, School of Mechanical Science and Engineering, HUST, Wuhan 430074, China 